\title{
Progress in Understanding the Toxicity of Gasoline and Diesel Engine Exhaust Emissions
}

Kristen J. Nikula, Gregory L. Finch, Richard A. Westhouse, JeanClare Seagrave and Joe L. Mauderly

Lovelace Respiratory Research Institute

Douglas R. Lawson National Renewable Energy Laboratory

Michael Gurevich U.S. Department of Energy 
SAE routinely stocks printed papers for a period of three years following date of publication. Direct your orders to SAE Customer Sales and Satisfaction Department.

Quantity reprint rates can be obtained from the Customer Sales and Satisfaction Department.

To request permission to reprint a technical paper or permission to use copyrighted SAE publications in other works, contact the SAE Publications Group.

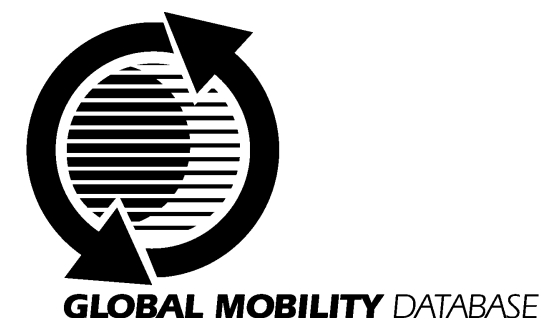

AII SAE papers, standards, and selected books are abstracted and indexed in the Global Mobility Database

\section{ISSN 0148-7191}

Positions and opinions advanced in this paper are those of the author(s) and not necessarily those of SAE. The author is solely responsible for the content of the paper. A process is available by which discussions will be printed with the paper if it is published in SAE Transactions. For permission to publish this paper in full or in part, contact the SAE Publications Group.

Persons wishing to submit papers to be considered for presentation or publication through SAE should send the manuscript or a 300 word abstract of a proposed manuscript to: Secretary, Engineering Meetings Board, SAE.

\section{Printed in USA}




\title{
Progress in Understanding the Toxicity of Gasoline and Diesel Engine Exhaust Emissions
}

\author{
Kristen J. Nikula, Gregory L. Finch, Richard A. Westhouse, JeanClare Seagrave and \\ Joe L. Mauderly \\ Lovelace Respiratory Research Institute \\ Douglas R. Lawson \\ National Renewable Energy Laboratory \\ Michael Gurevich \\ U.S. Department of Energy
}

\section{ABSTRACT}

To help guide heavy vehicle engine, fuel, and exhaust after-treatment technology development, the U.S. Department of Energy and the Lovelace Respiratory Research Institute are conducting research not addressed elsewhere on aspects of the toxicity of particulate engine emissions. Advances in these technologies that reduce diesel particulate mass emissions may result in changes in particle composition, and there is concern that the number of ultrafine $(<0.1$ micron) particles may increase. All present epidemiological and laboratory data on the toxicity of diesel emissions were derived from emissions of oldertechnology engines. New, short-term toxicity data are needed to make health-based choices among diesel technologies and to compare the toxicity of diesel emissions to those of other engine technologies. This research program has two facets: 1) development and use of short-term in vitro and in vivo toxicity assays for comparing the toxicities of gasoline and diesel exhaust emissions; and 2) determination of the disposition of inhaled ultrafine particles deposited in the lung. Responses of cultured cells, cultured lung slices, and rodent lungs to various types of particles were compared to develop an improved short-term toxicity screening capability. To date, chemical toxicity indicators of cultured human A549 cells and early inflammatory and cytotoxic indicators of rat lungs have given the best distinguishing capability. A study is now underway to determine the relative toxicities of exhaust samples from in-use diesel and gasoline engines. The samples are being collected under the direction of the National Renewable Energy Laboratory with support from DOE's Office of Heavy Vehicle Technologies. The ability to generate solid ultrafine particles and to trace their movement in the body as particles and soluble material was developed. Data from rodents suggest that ultrafine particles can move from the lung to the liver in particulate form. The quantitative disposition of inhaled ultrafine particles will be determined in rodents and nonhuman primates.

\section{INTRODUCTION}

Under a cooperative agreement with the Office of Heavy Vehicle Technologies, U.S. Department of Energy, the Lovelace Respiratory Research Institute is conducting research and development to provide health-related guidance for heavy vehicle engine, fuel, and exhaust after-treatment technology development. Advances in engine design, fuels, and exhaust after-treatment technologies aimed at reducing diesel particulate mass and nitrogen oxides have changed the nature of emissions. However, existing epidemiological and laboratory animal data on the toxicity of diesel particulate emissions are derived from exposures to emissions from older-technology engines. It is possible for changes in emissions to result in either greater or lesser toxicity. For example, it has been suggested that emission of increased numbers of ultrafine $(<0.1$ micron) particles of organic condensate may accompany the reduction of elemental carbon particles (Bagley, 1996), yet the health impact of such an increase in ultrafine particles is unknown. Thus, health information is needed to help guide choices among strategies to increase engine efficiency and reduce emissions. Furthermore, few data allow the health risks from diesel emissions to be directly compared to risks from engines burning gasoline and other fuels.

The current effort at Lovelace has two facets: 1) development and use of standardized short-term in vitro (using cultured cells and tissues) and in vivo (using intact animals) toxicity assays for comparing the toxicities of particulate emissions; and 2) evaluation of the disposition (clearance, penetration, translocation to other organs, and excretion) of inhaled ultrafine particles 
deposited in the respiratory tract. Standardized rapid assays of biological activity are needed because it is not practical to conduct long-term animal studies of the influence of each change in diesel technology on the toxicity of the resulting emissions, and epidemiological studies of newer emissions will not be possible for many years. Ultrafine particles are thought to penetrate lung tissues more rapidly, translocate to other organs to a greater degree, and have greater toxicity per unit of mass than larger fine particles, but existing information is largely anecdotal. There are no quantitative data on the distribution of ultrafine particles after deposition in the lung. Thus, improved short-term comparative toxicity assays and new information on the disposition of ultrafine particles are needed to estimate the potential health implications of the different diesel technologies and to compare the toxicity of petroleum diesel emissions to those of other engine and fuel technologies.

\section{DEVELOPMENT OF SHORT-TERM TOXICITY ASSAYS}

RATIONALE FOR APPROACH - Exposure of animals via inhalation is usually considered the most reliable method for evaluating the in vivo toxicity of airborne materials. However, inhalation studies resulting in substantial exposures take months to years to conduct and cost hundreds of thousands of dollars per sample. Therefore, alternative assays that provide early indicators of comparative toxicity are needed to evaluate potential health effects of changes in emissions in a timely, costeffective manner. Responses in these assay systems will not be followed to chronic health outcomes and will not be predictive of specific human diseases. However, the assays will be standardized so that a variety of samples can be directly compared, and they will measure endpoints (e.g., cell injury and inflammation) generally accepted to indicate toxicity and adverse health effects.

Short-term toxicity assays based on cultured cells and instillation of material into rodent lungs have been used primarily to explore mechanisms of toxicity of different materials. There has not been a concerted effort to compare the value of these systems for demonstrating subtle differences in toxicity among samples of generally similar toxicity. Cultured slices of organs have been used for similar purposes (Monteil et al., 1997), but there has been little validation of the utility of lung slices as an assay of particle toxicity. This project explored the relative values of a cultured epithelial cell line of human lung origin (A549), cultured rat lung slices, and instillation of material into intact rat lungs for determining gradations of toxicity among particle samples. The cell line has the advantage of using a single, standardized cell type that is readily available. Use of a cell line allows multiple samples and multiple doses to be assayed simultaneously under controlled conditions that minimize variability in responses due to factors other than the inherent toxicity of the samples. However, the cell line is the least representative of the actual complex lung tissue among the three assays. Responses that require interactions of two or more cell types cannot be measured in the cell culture system. Nevertheless, use of conditioned medium from particle-exposed macrophages to treat the epithelial cells may provide a means of examining some of these interactions (Barrett et al., 1998). The lung slices have the advantage of containing several cell types organized normally into tissues, but are technically complex to manage and have the smallest amount of previous information among the assays. Instillation into rodent lungs is the most realistic biological system for measuring lung responses among the three assays. Responses requiring complex interactions among multiple cell types can be measured. Instillation does not exactly duplicate inhalation. However, the small amount of material in each exhaust sample precludes the generation of sufficiently concentrated atmospheres for an adequate time to test these samples by inhalation. In this case, instillation provides a reasonable alternative to inhalation. One disadvantage to instillation compared to cell culture is that the use of live animals and the complexity of the measured responses tend to introduce greater variability that could make subtle differences in toxicity between samples difficult to detect.

METHODS - The variables explored included the best markers of toxicity and the optimum sampling time for each assay, and the ability of the assays to discriminate among different test particles comprising a range of toxicity. A key measure of toxicity for all the assays was the concentration of several different chemical signals elaborated by cells either during normal metabolism or in response to challenge with foreign material. These agents are thought to include some of the earliest and most sensitive signals of cellular responses to toxic materials. Some of these chemical signals, such as protein synthesis or adenosine triphosphate (ATP) content, indicate the general health of the cells. Others, commonly termed "cytokines" constitute the chemical signaling among cells for activation of defense or repair mechanisms, such as recruitment of inflammatory cells from the blood, or stimulation of cell division and growth. These cytokines included interleukin-8 (IL-8), growthregulated oncogene alpha (GRO-a), macrophage inflammatory protein-2 (MIP-2), and tumor necrosis factor alpha (TNF-a). Measurements also included release of the cytoplasmic enzyme lactate dehydrogenase $(\mathrm{LDH})$. This enzyme is present in the cytoplasm of lung cells, and its presence in the lung lining fluid is a reflection of severe cell injury and death (Henderson, 1991). These agents were measured in the culture medium for cell and lung tissue cultures, and in the bronchoalveolar lavage (lung washing) fluid of instilled lungs. In the cultured cell model, the cells remaining attached to the plate were assessed as a measure of loss of cell functional integrity, another indicator of toxicity. For instilled lungs, the number and type of inflammatory cells entering the lungs, leakage of 
protein into the lung as an indicator of inflammation and loss of normal tissue integrity, and the histopathology of the lungs were also evaluated.

A range of particle types was used for these studies, with the goal of comparing the responses of the assays to particles of differing type and toxicity. Diesel soot particles (DEPs) were scraped from filters collected from General Motors LH6 6.2 L V-8 engines burning EPA certification fuel and operated on the FTP light-duty cycle at the Institute. The carbon black (CB) was Elftex-12 furnace black obtained from Cabot. Both the DEP and CB would have been very similar, if not identical, to the materials used at Lovelace in a 2-year inhalation carcinogenesis bioassay of rats (Nikula et al., 1995). Residual oil fly ash (ROFA), obtained from the heat exchange section of the Boston Edison Mystic River power plant, was provided by Dr. John Godleski of Harvard University. Nickel subsulfide (Ni3S2), provided by Midwest Research Institute, was identical to material used at Lovelace in a 2-year inhalation carcinogenesis bioassay (U.S. NTP, 1994). Silica (Si) (5 mm Min-U-Sil) was obtained from U.S. Silica. Titanium dioxide (TiO2) was obtained from Fischer Scientific. The NIST diesel particulate matter (Standard Reference Material 1650) was obtained from the U.S. Department of Commerce National Institute of Standards and Technology (NIST; Gaithersburg, MD).

For each endpoint examined, one-way analysis of variance followed by an all pairwise multiple comparison procedure (Bonferroni's method) were used to determine if results differed significantly among groups. The level of statistical significance was set at $p<0.05$.

\section{RESULTS}

A549 Cell Culture - Toxicity and cellular responses to particle exposure were measured by several different parameters. Release of LDH, a measure of loss of membrane integrity, was significantly increased by a dose of $40 \mathrm{mg} / \mathrm{cm} 2$ of three particles considered highly cytotoxic (ROFA, Si, and Ni3S2) (Figure 1). However, at this dose, the other particles examined did not cause significant release of the enzyme. In another type of assay for toxicity, cells were plated in 96-well tissue culture clusters, exposed to particles at a range of concentrations, and the cells remaining adherent to the plates were stained with Crystal Violet. This assay showed loss of cells, and the results were comparable to release of LDH. However, because the sensitivity of this assay allowed it to be performed in the microtiter plate format, a range of doses could be tested simultaneously. At high doses, toxicity of the NIST diesel particulate matter was observed (Figure 2). ATP content was measured following exposure to $40 \mathrm{mg} / \mathrm{cm} 2$ of the various particles. Interestingly, when normalized to the protein content of the adherent cells, ROFA and Ni3S2 decreased the levels of ATP, but Si did not (Figure 3). Combined with the LDH and adherent cell data, these results suggest that $\mathrm{Si}$ caused a loss of adherent cells, but that the ATP content in cells remaining adherent was unaffected. Aside from overt toxicity, it is likely that exposure to toxic particles can affect expression of cytokines involved in recruitment of inflammatory cells. Figure 4 shows that $A 549$ cells exposed to $40 \mathrm{mg} / \mathrm{cm} 2$ of ROFA secreted significant amounts of IL-8; however, under these conditions, the assay did not distinguish among the other particles. Efforts are currently ongoing to assess the responses of these cells to a range of particle doses and of other cytokines. Finally, exposure to certain types of particulate material in vivo caused release of alkaline phosphatase to the epithelial lining fluid. Studies at this Institute (Henderson et al., 1995) have indicated that the source of this enzyme is most likely lung epithelial cells. Preliminary data show that exposure to particles caused release of alkaline phosphatase into the supernatant of A549 cell cultures. Strong responses were observed with two types of diesel particles and with ROFA (Figure 5). The decreased responses at high doses of particles are presumably due to cell death. However, the particles with the highest acute toxicity, $\mathrm{Si}$ and Ni3S2, had little effect on the release of this enzyme. These data suggest that there are several mechanisms by which particular material may cause alterations in cell viability and function. The results further indicate that this cellular model can provide important information on the biological effects of exhaust constituents.

Cultured Rat Lung Slices - After considerable effort to optimize and standardize the culture and particle treatment conditions for the assay, its responses to DEP, $\mathrm{CB}$, and ROFA were compared. The assay indicated a graded toxicity from $C B$ with the least toxicity to ROFA with the greatest. An example is given in Figure 6, which illustrates differences in secretion of MIP-2 by slices cultured for 11 days after instillation with $1 \mathrm{mg}$ particles/ $\mathrm{mL}$ incubation medium. (In the intact animal, MIP-2 recruits inflammatory cells.) Although the lung slice assay appeared to distinguish between DEP or ROFA and their corresponding controls, the control data varied considerably. This variability could make comparisons among particles or among experiments conducted at different times difficult to interpret. Also, the assay was more complex, time-consuming, and costly to perform than either cell culture or animal instillation. These experiments have been summarized by Westhouse et al. (1999).

Intratracheal Instillation in Intact Animals - Three types of particles were used to determine the sensitivity of the in vivo intratracheal instillation assay for distinguishing among particles of similar toxicity. The particles were DEP and CB as used in the two in vitro assays and a sample of diesel soot (DS) scrapped from filters collected from 1980 Model 5.7 L Oldsmobile V-8 engines. Nineweek-old male F344 rats were anesthetized with halothane, a cannula was placed in the trachea via the mouth, and $3 \mathrm{mg}$ of particles suspended in $0.5 \mathrm{~mL}$ saline was instilled into the lower trachea. The rats recovered, 
then groups of five were killed at either $4,6,12$, or 24 hours or 1 or 4 weeks after instillation. The lungs were removed and lavaged using a common and wellstandardized technique (Henderson, 1991) via the trachea with saline, which was then analyzed for cytokines, LDH, protein, and inflammatory cells. Not all analyses were conducted at all times. Optimal times for the cytokine assays are at 4,12 , and 24 hours, whereas optimal times for the other assays are generally at 12 and 24 hours and 1 and 4 weeks after instillation. Although the ability of the rat instillation assay to distinguish among particles having slightly different, but generally low toxicity is still being explored, preliminary results suggest that the integrated response of intact animals may have greater discriminatory ability than the two in vitro assays. For example, Figure 7 illustrates the concentrations of the enzyme LDH in lavage fluid at 6 and 24 hours and 1 week after instillation of CB, DEP, or DS. The enzyme concentration was elevated above the saline-instilled control value by $C B$ at all three times, whereas the enzyme concentrations were elevated above control values by DS at 6 and 24 hours and by DEP at 1 week. The concentrations of LDH in lavage fluid from CBinstilled rats were greater than those from DEP-instilled rats at the two later time points. These findings are consistent with the results of an earlier long-term inhalation study (Nikula et al., 1995), which also indicated that this CB was slightly more toxic than this DEP per unit of material retained in the lung. The concentrations at 1 week indicated that $C B$ was more toxic to lung cells than DS. Figures 8 and 9 illustrate the secretion of MIP-2 and the recruitment of inflammatory cells, respectively, into the lung lining fluid of rats at different times after instillation of the three particles. Inflammation is a common early response to toxic materials and also a common feature of the early stages of several lung diseases. All three particles caused secretion of MIP-2 and recruitment of inflammatory cells. However, the onset of the cellular response was delayed in the dieselexposed compared to the CB-exposed rats. The magnitude of the MIP-2 and cellular responses differed by particle type such that the overall inflammatory response was significantly greater for $\mathrm{CB}$ than for DEP or DS.

SUMMARY OF DEVELOPMENT WORK - The results of this development work suggested that overall, intratracheal instillation into the lungs of intact rats followed by bronchoalveolar lavage and histopathology was likely to give the most reliable assay of relative particle toxicity. The A549 cell cultures may also provide a useful tool for rapid toxicity comparisons because multiple particles and doses can be efficiently compared at multiple times. Currently, dose-response and timeresponse relationships in the cell cultures are being examined further. In addition, the use of cultured lung macrophages is being explored. Macrophages are cells that are key to responses because they engulf particles and release cytotoxic and inflammatory mediators. At this time, it does not appear that the lung slice assay offers any advantage over cell culture or lung instillation. Compared to cell cultures and instillation, variability among controls, technical difficulties, and difficulty in interpreting results are greater in lung slices. The lung instillation assay has the advantage over the other assays of allowing the full interplay among the various biological responses present in intact individuals. Because the assay does not involve inhalation, the exposure route of concern, it is not likely to produce data useful for quantitative estimates of risk to humans. It appears that the lung instillation assay can, however, meet the need for a rapid, low-cost assay of relative toxicity of particles in the lung, and especially if materials known to induce a positive response and standard control particles, such as the NIST diesel soot, are included in each assay.

APPLICATION OF THESE MODELS - In the future, the rapid assays will be used to rank toxicity of emissions from a matrix of exhaust particles and semi-volatile organic compounds (SVOCs). This matrix will include diesel and gasoline emissions. The samples will be taken from older and newer technology diesels, and from average, high emitter, and "smoker" gasoline engines. Dynamometer samples as well as traffic tunnel samples representing diesel or gasoline fleets will be evaluated. The goal is to provide information helping to place the comparative health risks from airborne emissions from diesel and gasoline engine technologies in perspective.

The samples, which will consist of combined particulate matter and SVOCs, are being collected under a contract administered by Dr. Douglas Lawson of the National Renewable Energy Laboratory. The samples will be coded prior to being supplied to Lovelace investigators. They will evaluate the toxicity of the samples using a standardized combination of the cell culture and intratracheal instillation assays described above. In addition, mutagenicity will be determined using the Ames Assay, in which mutations are observed in Salmonella bacteria. Although bacterial mutagenicity cannot be extrapolated directly to cancer risk, the test is widely accepted for comparative screening purposes. Mutagenicity will be determined using two strains of $S$. typhimurium (TA98 and TA100) with and without metabolic activation achieved by adding Aroclor 1254induced rat liver S-9 microsomal fraction. All assays will be done in triplicate, and positive control compounds will be evaluated concurrently.

For the cell culture studies, cells will be incubated with the samples at a range of concentrations in the culture media. Parallel cell cultures (controls) will be treated with only the appropriately diluted solvent used in the provided samples. In addition, all assays will include cells incubated with the NIST diesel soot sample as a way to standardize or calibrate the responses across assays. Lastly, all assays will include cells incubated with endotoxin because it is known to activate cells so they release several mediators that will be measured (positive control). Endpoints will include cell adhesion to the 
substrate, protein and ATP content of cells, LDH release, IL-8 release, GRO-a release, and alkaline phosphatase activity in culture supernates. Dose-response relationships will be graphed and statistically compared among samples.

For the intratracheal instillation studies, rats will be instilled with either of two doses of the sample or with the appropriately diluted solvent and instillation vehicle as a control. In addition, rats will be instilled with the NIST soot sample as a way to standardize or calibrate the responses across assays. Lastly, all assays will include rats instilled with endotoxin because it activates cells in the lungs so they release several mediators that will be measured (positive control). Groups of five rats per particle type and dose, or controls, will be killed at several times ranging from 4 hours to 4 weeks after instillation. Lung lavage cells and fluid will be collected for analyses, and lung tissue will be fixed for histopathology. The following parameters will be evaluated at the appropriate times after instillation (not all parameters will be evaluated at each time). Total cell counts, differential cell counts, and protein from lavage will be used to quantify the magnitude and character of the intra-alveolar inflammatory cell and exudative response. LDH will be used as an indicator of cell permeability due to cytotoxicity or death, whereas b-glucuronidase enzyme activity will be used as an indicator of increased macrophage activity or lysis. Superoxide release from lavage macrophages and neutrophils will be measured because the mechanisms of lung injury after exposure to particles have been postulated to involve oxidant generation, and the generation of reactive oxygen species is thought to be an essential step in activating the release of chemical signals. TNF-a and MIP-2, which are important to cell activation and inflammatory cell recruitment, will be measured in lavage fluid. Lastly, histopathology will be used to assess the magnitude, character, and location of the inflammatory response as well as epithelial responses to the particles or the inflammation.

\section{DISPOSITION OF INHALED ULTRAFINE PARTICLES}

The work on ultrafine particle disposition continues, and definitive results are just becoming available. This effort required considerable technical development to enable the detection and quantitation of very small amounts of tiny particles dispersed in various tissues and fluids. The lack of detection ability has largely been responsible for the lack of previous work in this area. Another major difficulty due to the high surface area to mass ratio of ultrafine particles is ensuring that the material does not dissolve before it is measured in the body. Our research approach was to: 1) produce radiolabeled particles that could be aerosolized in uniform sizes; 2) determine the time course of the dissolution of the particles; and 3) measure the time course of the distribution of particles in rats and monkeys. Work to date has focused on the use of radiolabeled silver particles $(110 \mathrm{mAg})$, because of our previous experience in generating this material in the appropriate size range and because $110 \mathrm{mAg}$ in small quantities should be relatively inert in the lung.

The $110 \mathrm{mAg}$ particles were generated by heating radiolabeled foil and allowing the vapor to condense into particles under controlled cooling and dilution conditions. After trials defining the conditions necessary to reproducibly generate $110 \mathrm{mAg}$ in fine and ultrafine sizes, the solubility of the particles in simulated body fluid was tested using a dialysis bag system. These results indicated that a sufficient portion of the material would remain in particulate form to evaluate disposition in the body during times up to a few days after inhalation.

An initial inhalation study in rats has been done, and the results are being analyzed. Twelve-week-old male F344 rats were exposed by inhalation to $110 \mathrm{mAg}$ particles having a count median diameter of 20 nanometers. Rats received an initial body burden (IBB) of approximately $130 \mathrm{kBq}$ at a deposited particle mass of $<1 \mathrm{ng}$ from the 35-minute exposure. Immediately thereafter, then at 2, 6, 24 , and 72 hours after exposure, groups of four rats were sacrificed to quantify the $110 \mathrm{mAg}$ in selected tissues. The $110 \mathrm{mAg}$ cleared rapidly from the lung, with only $60 \%$ and $27 \%$ of the IBB remaining at 24 and 72 hours, respectively. Most of the $110 \mathrm{mAg}$ excreted from the body was eliminated through the feces. As a percentage of the sacrifice body burden, the activity in lungs was $28 \%$, $10 \%$, and $2 \%$ at 6,24 , and 72 hours after exposure, respectively; the activity in the Gl tract was $56 \%$ and $50 \%$ at 24 and 72 hours, respectively; and the activity in liver was $10 \%$ and $7 \%$ at 24 and 72 hours, respectively. A tissue digestion and ultracentrifugation procedure was used to determine if the remaining $110 \mathrm{mAg}$ was in particulate or dissolved form. Preliminary data indicate that the material was primarily in particulate form in the lung and liver, and in solubilized form in the blood. Tissue sections were evaluated by autoradiography and electron microscopy to visualize the particles and their location. In the lung, particles were present extracellularly in the lining fluid, in alveolar macrophages, extracellularly in the interstitium, and in interstitial macrophages. In the liver, particles appeared to be located in hepatocytes, especially in the centrolobular portion of the liver 24 and 72 hours after inhalation. These results have been summarized by Finch et al. (1999).

The initial results confirm the fact that a significant portion of poorly soluble ultrafine particles leaves the lung rapidly after deposition. Pending completion of the rat study and conclusion that the $110 \mathrm{mAg}$ inhalation technique provides acceptable data, a similar study will be performed using monkeys. Questions have been raised about the potential toxicity of ultrafine particulate emissions, and some have suggested that the majority of the ultrafine particulate material in emissions from new diesel engines consists of organic condensate. If this suggestion is proven by experts in emissions analyses, the next step will be to evaluate the behavior of ultrafine 
organic particles after inhalation. At this time, it is not known if they will behave as particles or as vapors because of their rapid solubility. Together, these results should provide a much-improved understanding of the behavior of ultrafine particles after deposition in the lung by inhalation.

\section{ACKNOWLEDGEMENT}

Research supported by DOE Office of Biological and Environmental Research and Office of Energy Efficiency and Renewable Energy under Cooperative Agreement number DE-FC04-96AL76406.

\section{REFERENCES}

1. Bagley, S.T., "Characterization of Fuel and AfterTreatment Device Effects on Diesel Emissions," Health Effects Institute Report No. 76, 1996.

2. Barrett, E.G., C. Johnston, G. Oberdörster, and J.N. Finkelstein, "Silica-induced Chemokine Expression in Alveolar Type II Cells is Mediated by TNF-alpha. Am. J. Physiol. 275, L1110-1119, 1998.

3. Finch, G.L., K.J. Nikula, E.B. Barr, J.C. Seagrave, M.B. Snipes, and J.L. Mauderly, "Biokinetics of an Inhaled Ultrafine Silver Aerosol in Rats," The Toxicologist 48, 134 (abstract), 1999.

4. Henderson, R.F., "Analysis of Respiratory Tract Lining Fluids to Detect Injury," 1990 Focus on Pulmonary Pharmacology and Toxicology, $M$. Hollinger, Ed., Vol. II, pp. 1-18, CRC Press, Boca Raton, 1991.

5. Henderson, R.F., G.G. Scott, and J.J. Waide, "Source of Alkaline Phosphatase Activity in Epithelial Lining Fluid of Normal and Injured F344 Rat Lungs," Toxicol. Appl. Pharmacol. 134, 170-174, 1995.

6. Monteil, C., F. Fouquet, M. Guerbert, J.M. Jounay, and J.P. Morin, "The Use of Precision-Cut Rat Lung Slices as an In Vitro Tool in Toxicology: Effects of Diesel Exhaust," The Toxicologist 36, 327 (abstract), 1997.

7. Nikula, K.J., M.B. Snipes, E.B. Barr, W.C. Griffith, R.F. Henderson, and J.L. Mauderly, "Comparative Pulmonary Toxicities and Carcinogenicities of Chronically Inhaled Diesel Exhaust and Carbon Black in F344 Rats," Fundam. Appl. Toxicol. 25, 8094, 1995.

8. U.S. National Toxicology Program, "Toxicology and Carcinogenesis Studies of Nickel Subsulfide (CAS No. 12035-72-2) in F344 Rats and B6C3F1 Mice (Inhalation Studies)," TR-453, Research Triangle Park, NC, 1994.

9. Westhouse, R.A., F.F. Hahn, A.H. Rebar, and N.F. Johnson, "The Relative Toxicity of Particles in Rat Lung Tissue Slice Cultures," The Toxicologist 48, 66 (abstract), 1999. 


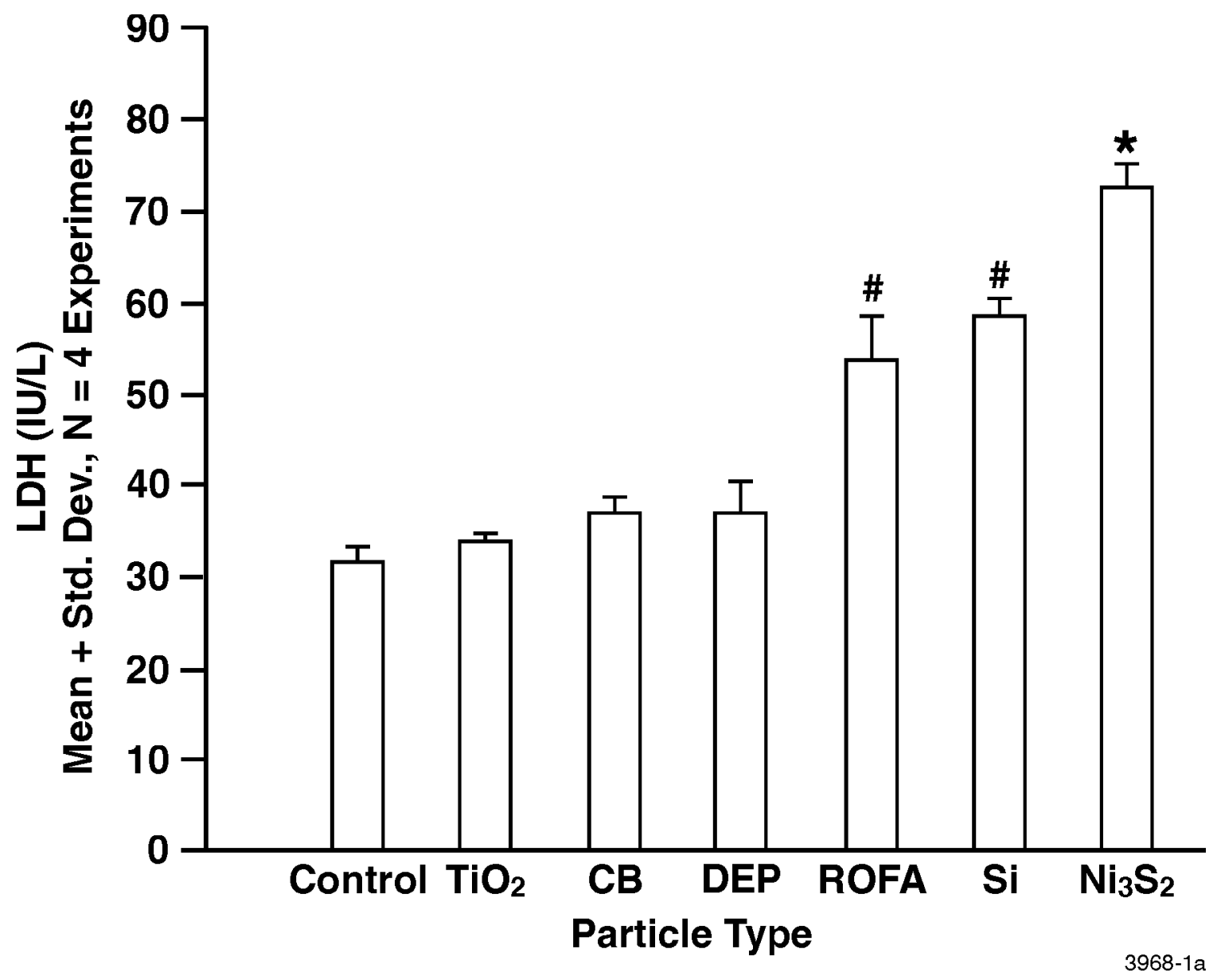

Figure 1. LDH concentration in A549 cell culture media after incubation for 20 hours with six types of particles or no particles (control) and analyzed 18 hours later. ${ }^{*}=$ significantly different from control and all other particles. \# = significantly different from control, $\mathrm{TiO}_{2}, \mathrm{DEP}, \mathrm{CB}, \mathrm{Ni}_{3} \mathrm{~S}_{2}$. 


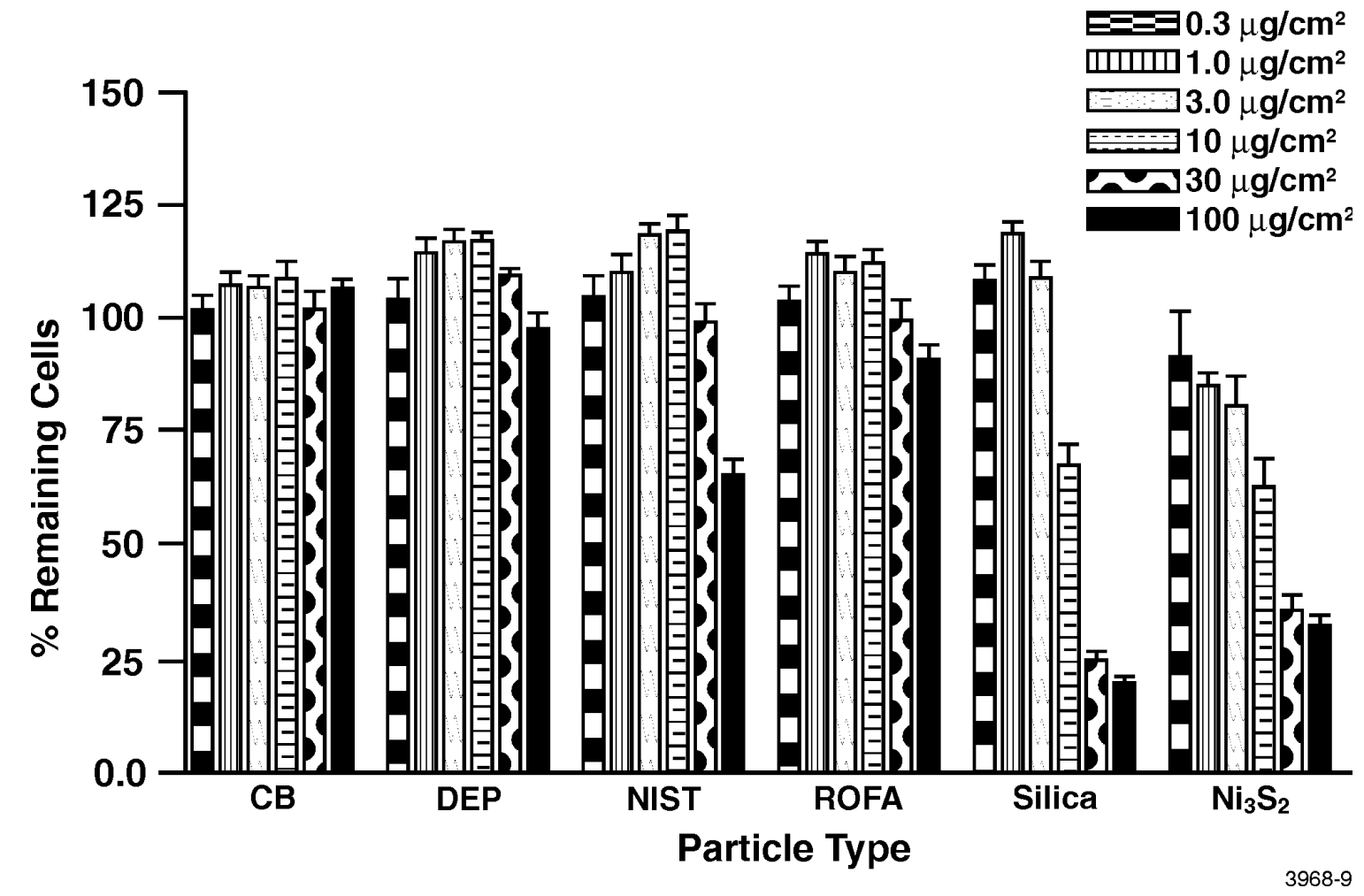

Figure 2. Adherent cells detected by Crystal Violet staining after incubation of A549 cells with six types of particles at various concentrations for 20 hours. Histograms represent means plus standard deviations, $n=$ four experiments. 


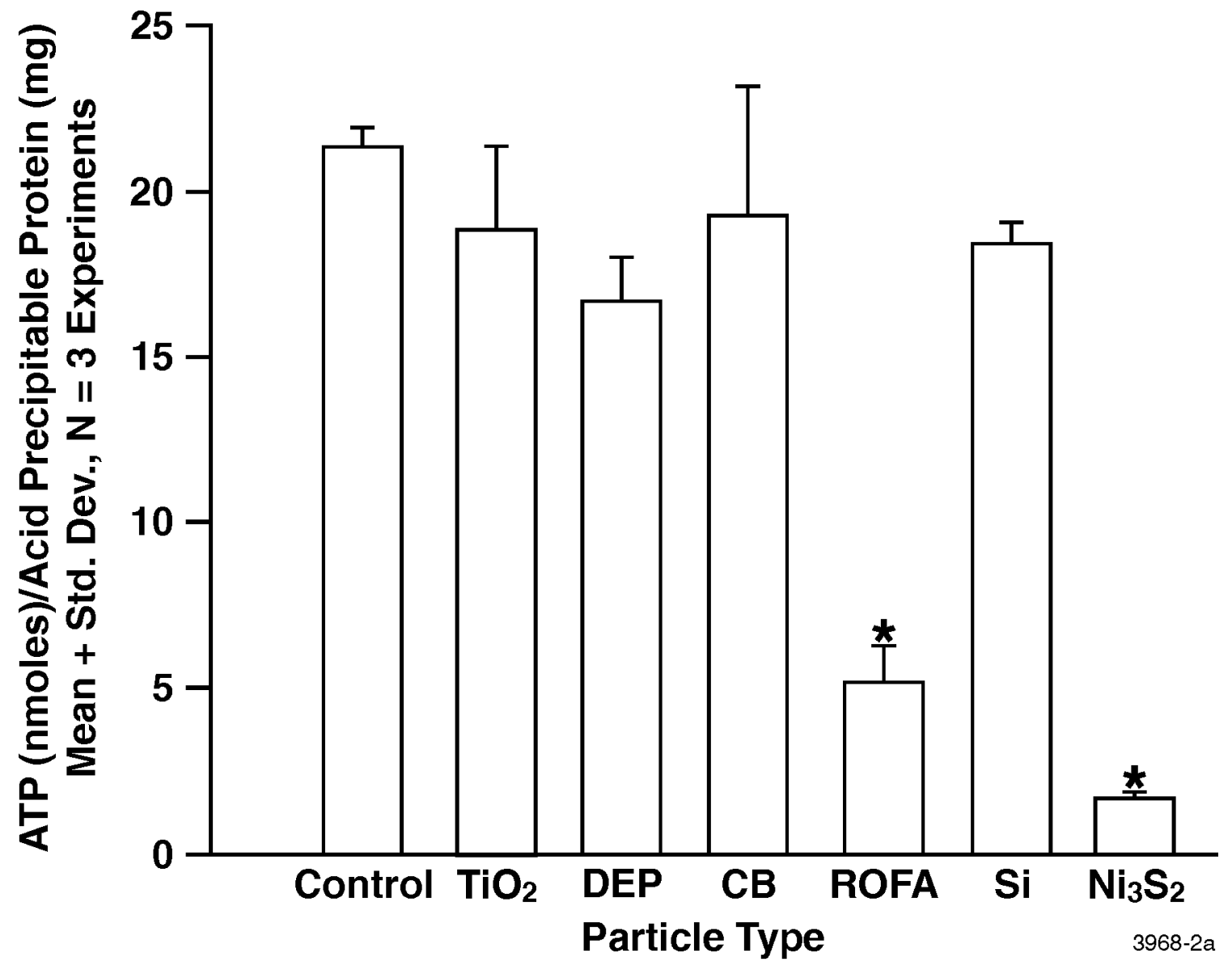

Figure 3. ATP content of A549 cells incubated with six types of particles or no particles (control) for 20 hours. ${ }^{*}$ = significantly different from control and all other particles. 


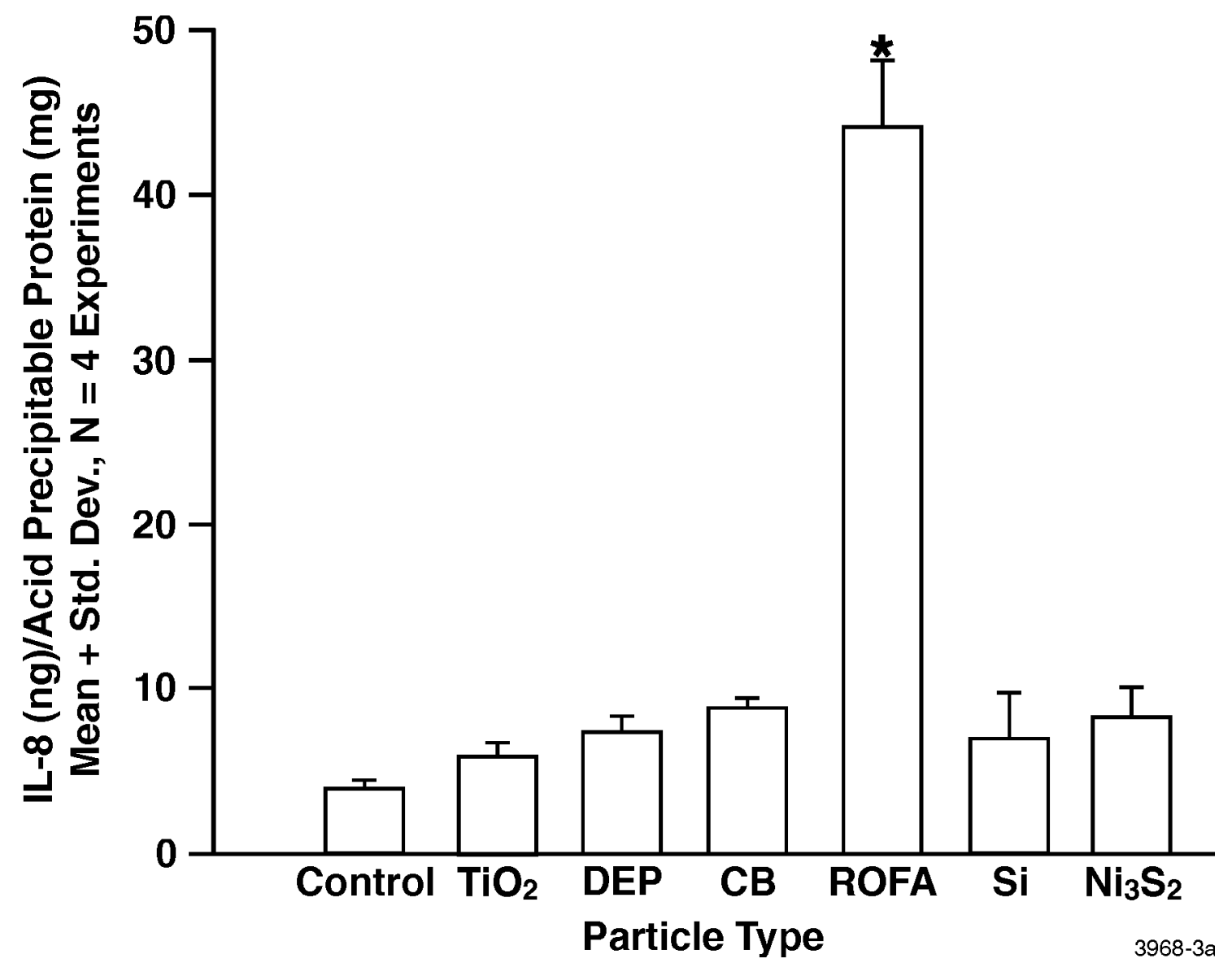

Figure 4. IL-8 secretion of A549 cells incubated with six types of particles or no particles for 20 hours. Values are normalized by total acid precipitable protein. ${ }^{*}=$ significantly different from control and all other particles.

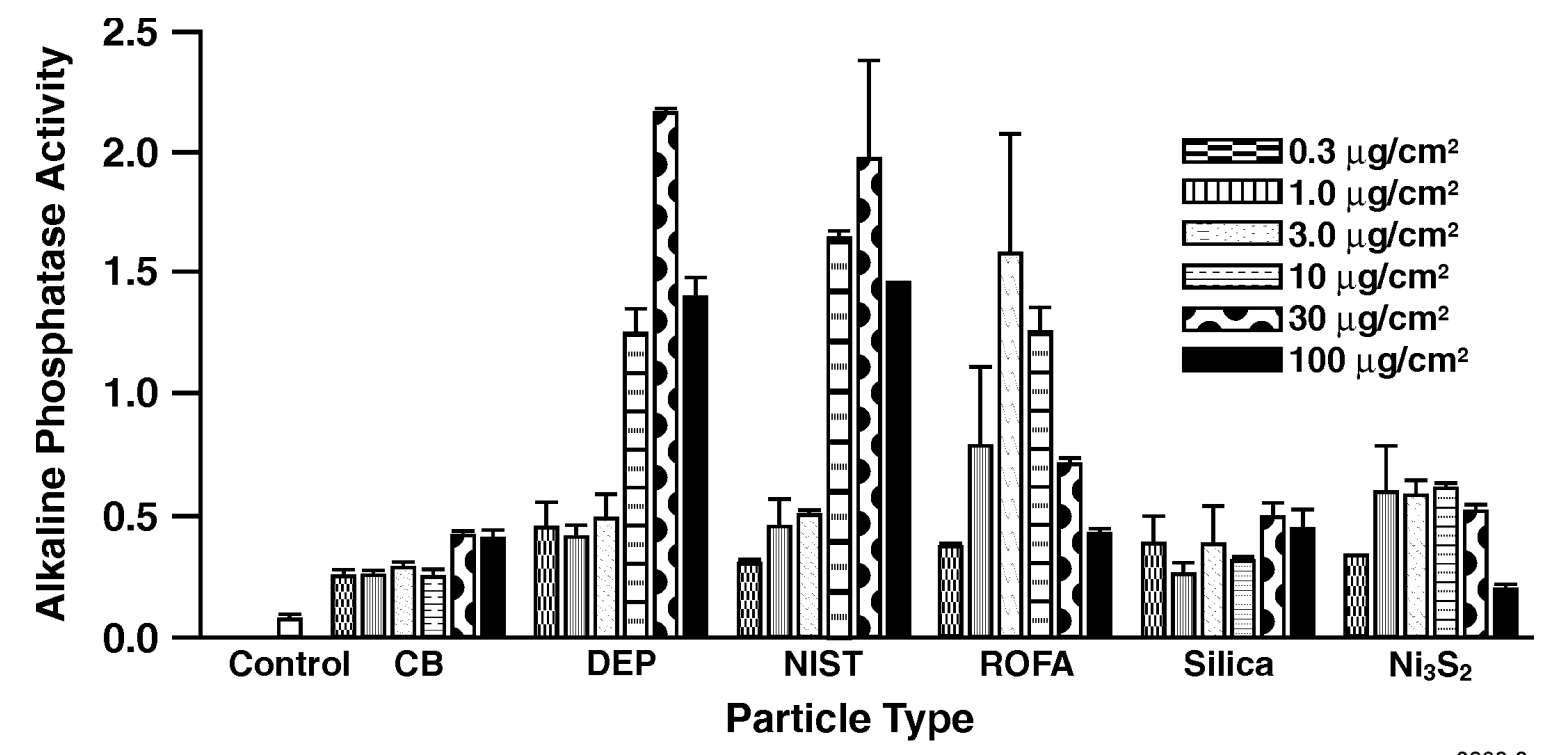

Figure 5. Alkaline phosphatase activity in supernatants from A549 cells cultured with six types of particles or no particles 


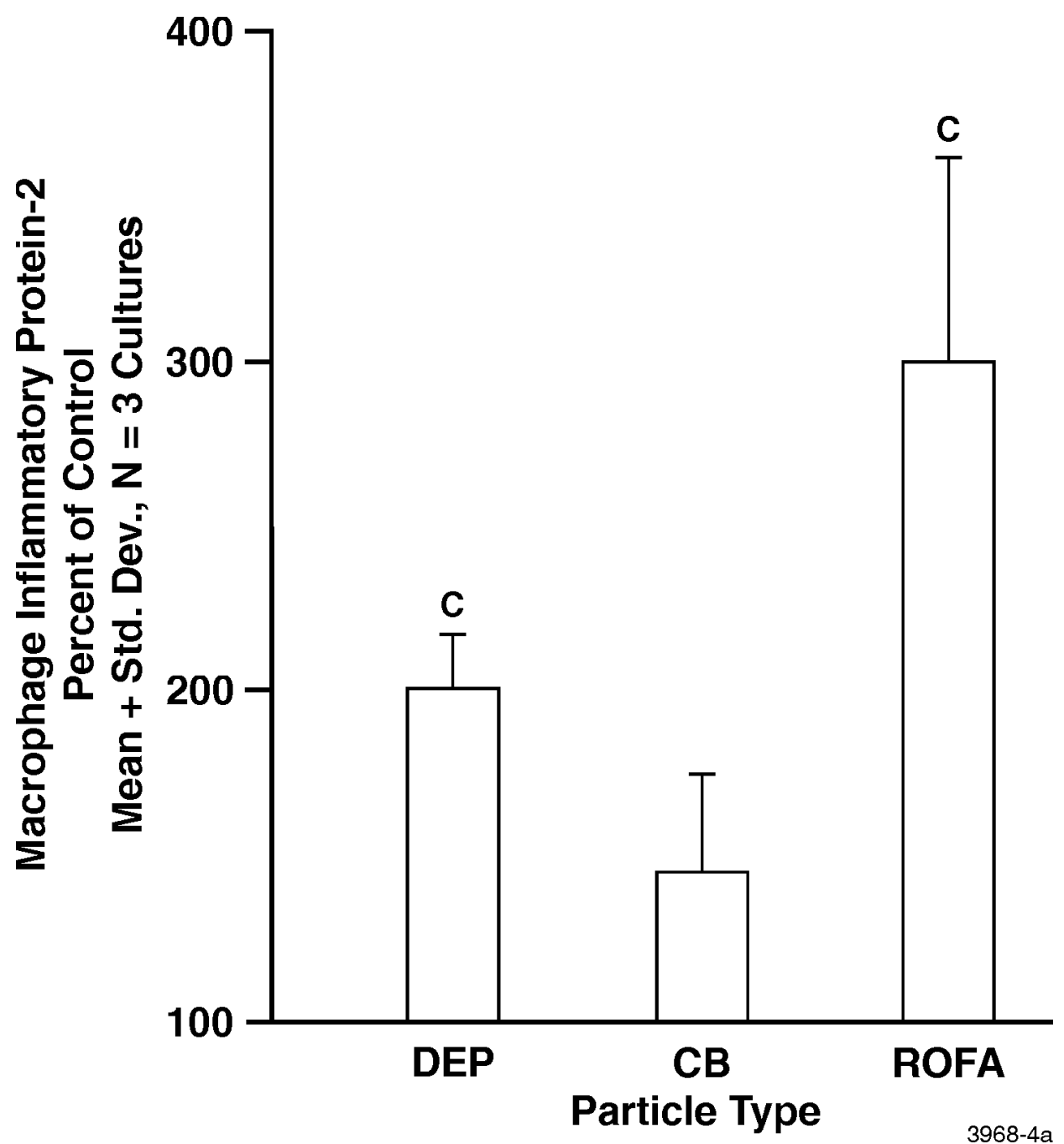

Figure 6. Secretion of MIP-2 by rat lung slice cultures after 11 days of incubation with three types of particles. MIP-2 values were normalized to total acid precipitable protein. The data are means and standard deviations of three cultures for each particle, expressed as percentages of the mean control, or untreated, response. At top of histogram, $\mathrm{c}=$ significantly different from control. 


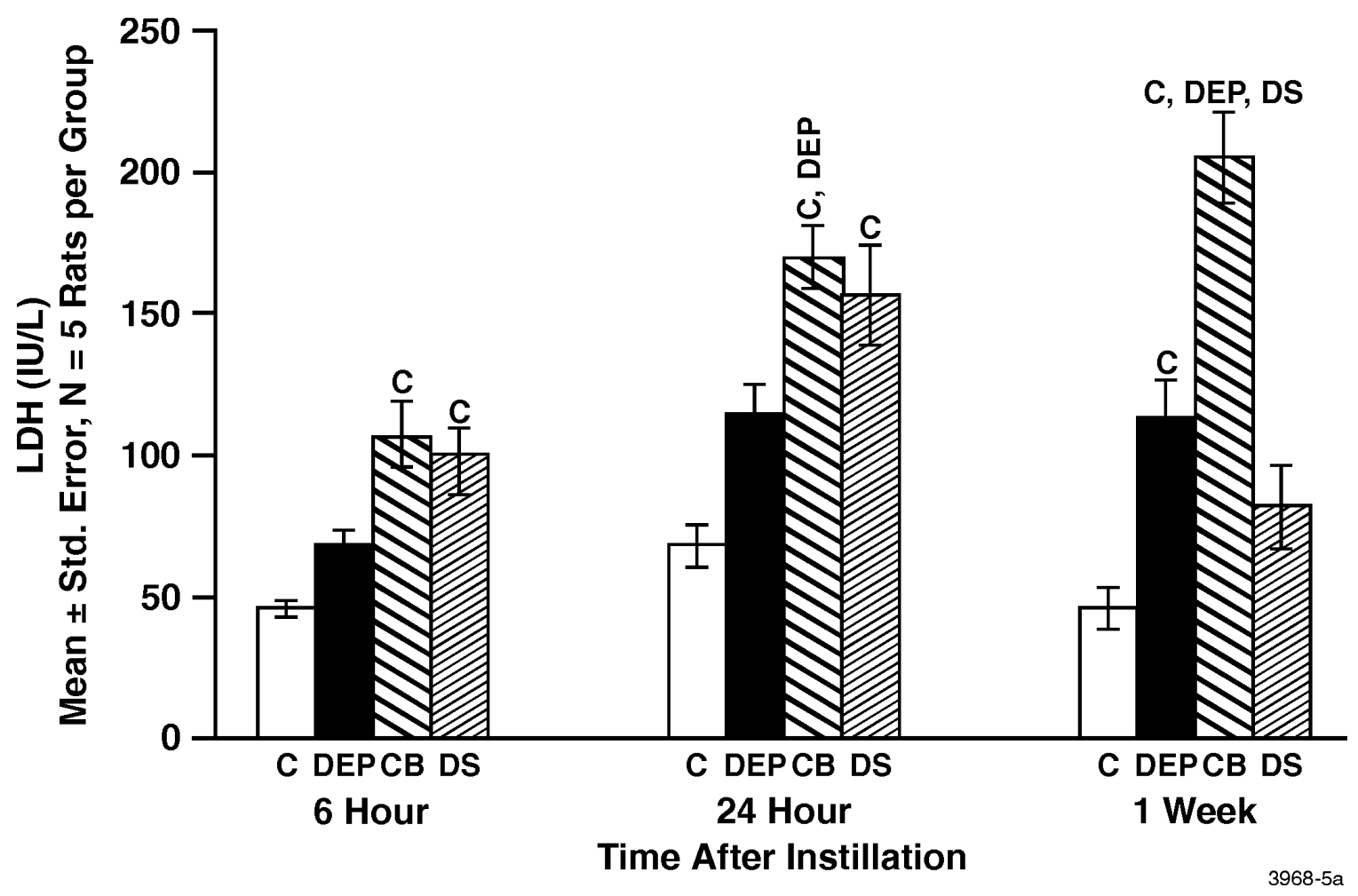

Figure 7. LDH concentration in lavage fluid of rats sacrificed at different times after intratracheal instillation of saline (control) or $3 \mathrm{mg}$ of DEP, CB, or DS. Particle or control (c) designations at the top of each histogram indicate that the results are different from those for the designated particle type or control. For example, results for $\mathrm{CB}$ at 1 week are significantly different from those for control, DEP, and DS.

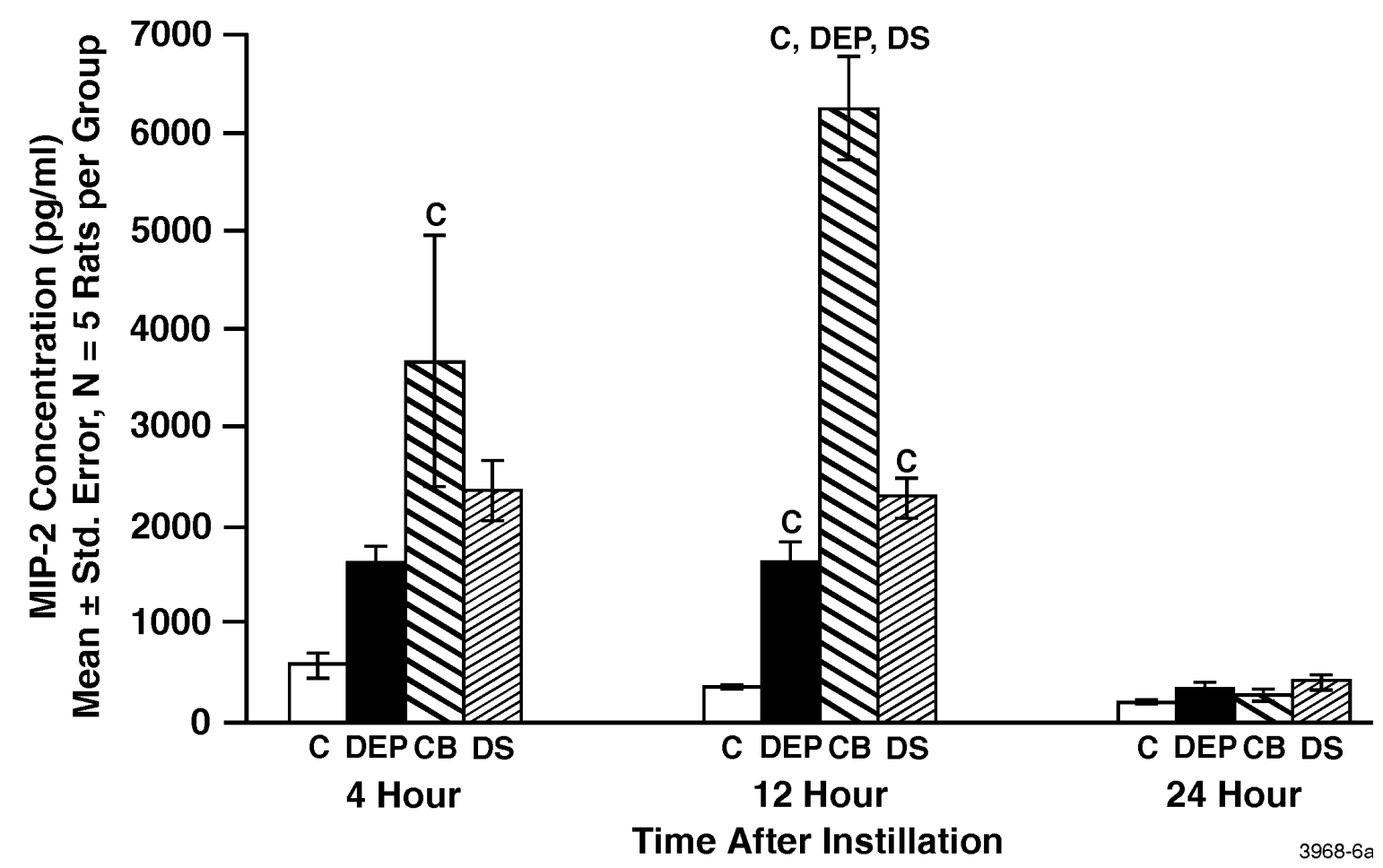

Figure 8. MIP-2 concentration in lavage fluid of rats sacrificed at different times after intratracheal instillation of saline (control) or $3 \mathrm{mg}$ of DEP, CB, or DS. Particle or control (c) designations at the top of each histogram indicate that the results are different from those for the designated particle type or control. For example, results for $\mathrm{CB}$ at 12 hours are significantly different from control, DEP, and DS. 


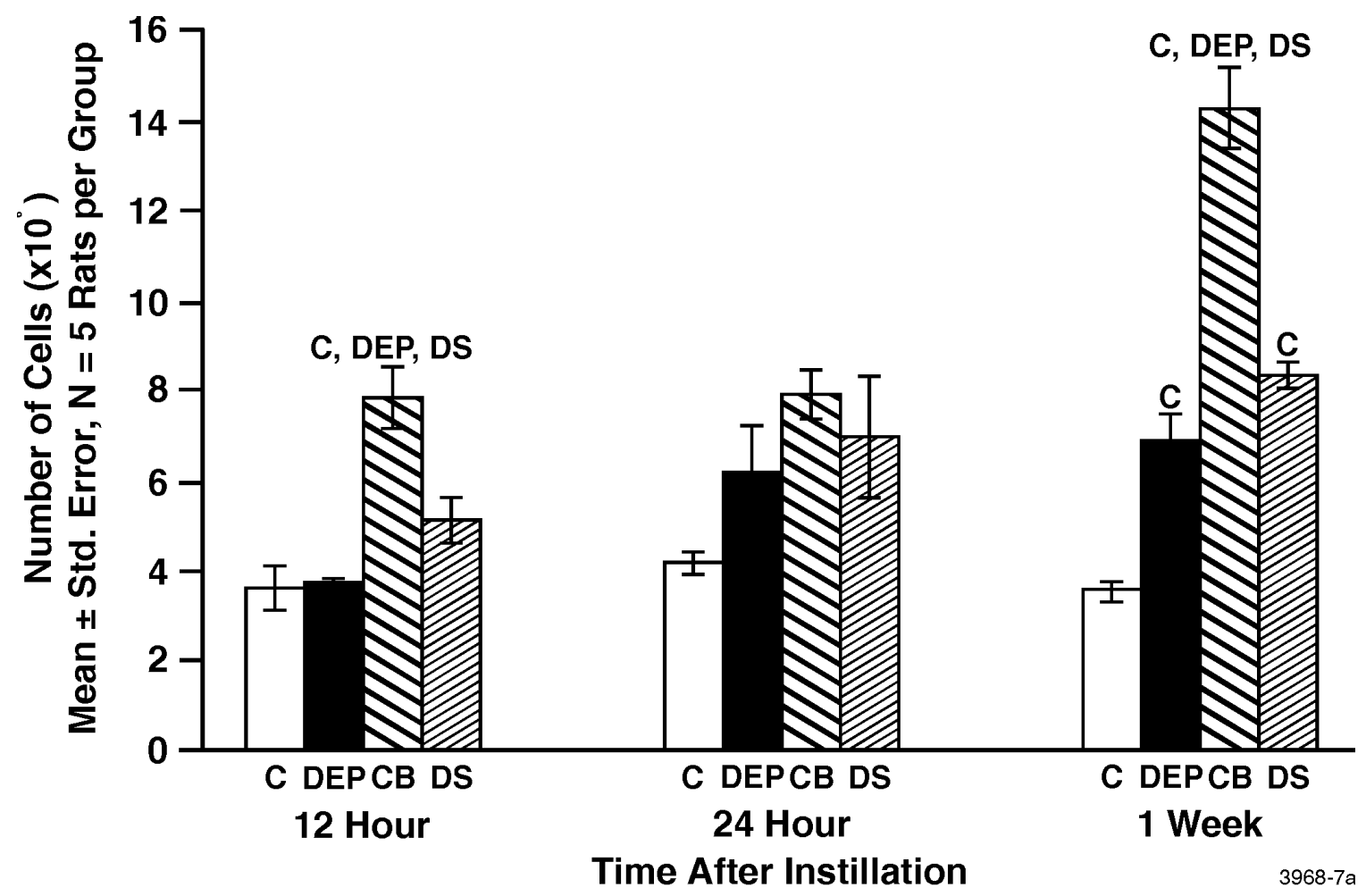

Figure 9. Total cells in lavage fluid of rats sacrificed at different times after intratracheal instillation of saline (control) or 3 $\mathrm{mg}$ of DEP, CB, or DS. Particle or control (c) designations at the top of each histogram indicate that the results are different from those for the designated particle type or control. For example, results for CB at 12 hours and 1 week are significantly different from control, DEP, and DS. 